\title{
NOTE ON THE TWO-COMPONENT ANALOGUE OF TWO-DIMENSIONAL LONG WAVE - SHORT WAVE RESONANCE INTERACTION SYSTEM
}

\author{
KEN-ICHI MARUNO \\ Department of Mathematics, The University of Texas-Pan American, Edinburg, TX 78541, USA \\ e-mail: kmaruno@utpa.edu \\ YASUHIRO OHTA \\ Department of Mathematics, Kobe University, Rokko, Kobe 657-8501, Japan \\ and MASAYUKI OIKAWA \\ Research Institute for Applied Mechanics, Kyushu University, Kasuga, Fukuoka, 816-8580, Japan
}

\begin{abstract}
An integrable two-component analogue of the two-dimensional long wave - short wave resonance interaction (2c-2d-LSRI) system is studied. Wronskian solutions of 2c-2d-LSRI system are presented. A reduced case, which describes resonant interaction between an interfacial wave and two surface wave packets in a two-layer fluid, is also discussed.
\end{abstract}

2002 Mathematics Subject Classification. 35Q51, 35Q55, 37K40

1. Introduction. In these past decades, vector soliton equations have received so much attention in mathematical physics and non-linear physics $[\mathbf{1}, \mathbf{2}, \mathbf{8}, \mathbf{1 3}]$. Recently, we derived the following system in a two-layer fluid using reductive perturbation method, which was motivated by a paper by Onorato et al. $[9,12]$ :

$$
\begin{gathered}
\mathrm{i}\left(S_{t}^{(1)}+S_{y}^{(1)}\right)-S_{x x}^{(1)}+L S^{(1)}=0, \quad \mathrm{i}\left(S_{t}^{(2)}-S_{y}^{(2)}\right)-S_{x x}^{(2)}+L S^{(2)}=0, \\
L_{t}=2\left(\left|S^{(1)}\right|^{2}+\left|S^{(2)}\right|^{2}\right)_{x} .
\end{gathered}
$$

This system is an extension of the two-dimensional long wave - short wave resonance interaction system $[\mathbf{1 4}, \mathbf{1 0}]$ and describes the two-dimensional resonant interaction between an interfacial gravity wave and two surface gravity packets propagating in directions symmetric about the propagation direction of the interfacial wave in a twolayer fluid.

In this paper, we will study this system and its integrable modification,

$$
\begin{gathered}
\mathrm{i}\left(S_{t}^{(1)}+S_{y}^{(1)}\right)-S_{x x}^{(1)}+L S^{(1)}=2 \mathrm{i} S^{(2)^{*}} Q, \\
\mathrm{i}\left(S_{t}^{(2)}-S_{y}^{(2)}\right)-S_{x x}^{(2)}+L S^{(2)}=2 \mathrm{i} S^{(1)^{*}} Q, \\
L_{t}=2\left(\left|S^{(1)}\right|^{2}+\left|S^{(2)}\right|^{2}\right)_{x}, \quad Q_{x}=S^{(1)} S^{(2)} .
\end{gathered}
$$

where * means complex conjugate. In our recent paper [11], we studied

$$
\begin{gathered}
\mathrm{i}\left(S_{t}^{(1)}+S_{y}^{(1)}\right)-S_{x x}^{(1)}+L S^{(1)}=0, \quad \mathrm{i}\left(S_{t}^{(2)}+S_{y}^{(2)}\right)-S_{x x}^{(2)}+L S^{(2)}=0, \\
L_{t}=2\left(\left|S^{(1)}\right|^{2}+\left|S^{(2)}\right|^{2}\right)_{x} .
\end{gathered}
$$


Note that this system is different from system (1) only in the sign of $y$-derivative term $S_{y}^{(2)}$.

2. Bilinear forms and Wronskian solutions. Consider a two-component analogue of two-dimensional long wave - short wave resonance interaction (2c-2dLSRI) system (2). Using the dependent-variable transformation $L=-(2 \log F)_{x x}$, $S^{(1)}=G / F, S^{(2)}=H / F, Q=-K^{*} / F$, we obtain

$$
\begin{array}{ll}
\left(D_{x}^{2}-\mathrm{i}\left(D_{t}+D_{y}\right)\right) G \cdot F=2 \mathrm{i} H^{*} K^{*}, & D_{x} D_{t} F \cdot F=-2\left(G G^{*}+H H^{*}\right), \\
\left(D_{x}^{2}-\mathrm{i}\left(D_{t}-D_{y}\right)\right) H \cdot F=2 \mathrm{i} G^{*} K^{*}, & D_{x} K \cdot F=-G^{*} H^{*} .
\end{array}
$$

These bilinear forms have the three-component Wronskian solution $[\mathbf{3}, \mathbf{4}, \mathbf{7}]$.

Consider the following three-component Wronskian:

$$
\tau_{N M L}=\left|\begin{array}{lll}
\varphi & \psi & \chi
\end{array}\right|,
$$

where $\boldsymbol{\varphi}, \boldsymbol{\psi}$ and $\chi$ are $(N+M+L) \times N,(N+M+L) \times M$ and $(N+M+L) \times L$ matrices, respectively: $\boldsymbol{\varphi}=\left(\partial_{x_{1}}^{j-1} \varphi_{i}\right)_{1 \leq i \leq N+M+L}^{1 \leq j \leq N}, \quad \boldsymbol{\psi}=\left(\partial_{x_{1}}^{j-1} \psi_{i}\right)_{1 \leq i \leq N+M+L}^{1 \leq j \leq M} \quad$ and $\chi=\left(\partial_{x_{1}}^{j-1} \chi_{i}\right)_{1 \leq i \leq N+M+L}^{1 \leq j \leq L}$, and $\varphi_{i}$ is an arbitrary function of $x_{1}$ and $x_{2}$ satisfying $\partial_{x_{2}} \varphi_{i}=\partial_{x_{1}}^{2} \varphi_{i}$, and $\psi_{i}$ and $\chi_{i}$ are arbitrary functions of $y_{1}$ and $z_{1}$, respectively. The above Wronskian satisfies

$$
\begin{aligned}
& \left(D_{x_{1}}^{2}-D_{x_{2}}\right) \tau_{N+1, M-1, L} \cdot \tau_{N M L}=0, \quad\left(D_{x_{1}}^{2}-D_{x_{2}}\right) \tau_{N+1, M, L-1} \cdot \tau_{N M L}=0, \\
& D_{x_{1}} D_{y_{1}} \tau_{N M L} \cdot \tau_{N M L}=2 \tau_{N+1, M-1, L} \tau_{N-1, M+1, L}, \\
& D_{x_{1}} D_{z_{1}} \tau_{N M L} \cdot \tau_{N M L}=2 \tau_{N+1, M, L-1} \tau_{N-1, M, L+1}, \\
& D_{x_{1}} \tau_{N, M+1, L-1} \cdot \tau_{N M L}=-\tau_{N-1, M+1, L} \tau_{N+1, M, L-1}, \\
& D_{y_{1}} \tau_{N-1, M, L+1} \cdot \tau_{N M L}=-\tau_{N, M-1, L+1} \tau_{N-1, M+1, L}, \\
& D_{z_{1}} \tau_{N+1, M-1, L} \cdot \tau_{N M L}=-\tau_{N+1, M, L-1} \tau_{N, M-1, L+1} .
\end{aligned}
$$

Setting

$$
\begin{array}{rlll}
f=\tau_{N M L}, & g=\tau_{N+1, M-1, L}, & h=\tau_{N-1, M, L+1}, & k=\tau_{N, M+1, L-1}, \\
\bar{g}=\tau_{N-1, M+1, L}, & \bar{h}=\tau_{N+1, M, L-1}, & \bar{k}=\tau_{N, M-1, L+1},
\end{array}
$$

we have the following bilinear forms:

$$
\begin{aligned}
& \left(D_{x_{1}}^{2}-D_{x_{2}}\right) g \cdot f=0, \quad\left(D_{x_{1}}^{2}+D_{x_{2}}\right) \bar{g} \cdot f=0, \quad D_{x_{1}} D_{y_{1}} f \cdot f=2 g \bar{g}, \\
& \left(D_{x_{1}}^{2}+D_{x_{2}}\right) h \cdot f=0, \quad\left(D_{x_{1}}^{2}-D_{x_{2}}\right) \bar{h} \cdot f=0, \quad D_{x_{1}} D_{z_{1}} f \cdot f=2 h \bar{h}, \\
& D_{x_{1}} k \cdot f=-\bar{g} \bar{h}, \quad D_{y_{1}} h \cdot f=-\bar{g} \bar{k}, \quad D_{z_{1}} g \cdot f=-\bar{h} \bar{k}, \\
& D_{x_{1}} \bar{k} \cdot f=g h, \quad D_{y_{1}} \bar{h} \cdot f=g k, \quad D_{z_{1}} \bar{g} \cdot f=h k .
\end{aligned}
$$

By the change of independent variables $x_{1}=x, x_{2}=-\mathrm{i} y, y_{1}=y-t, z_{1}=-y-t$ $(x, y, t:$ real $)$, we have $\partial_{x}=\partial_{x_{1}}, \partial_{y}=-\mathrm{i} \partial_{x_{2}}+\partial_{y_{1}}-\partial_{z_{1}}, \partial_{t}=-\partial_{y_{1}}-\partial_{z_{1}}$. Thus, we obtain

$$
\begin{array}{ll}
\left(D_{x}^{2}-\mathrm{i}\left(D_{t}+D_{y}\right)\right) g \cdot f=-2 \mathrm{i} \bar{h} \bar{k}, & \left(D_{x}^{2}+\mathrm{i}\left(D_{t}+D_{y}\right)\right) \bar{g} \cdot f=-2 \mathrm{i} h k, \\
\left(D_{x}^{2}-\mathrm{i}\left(D_{t}-D_{y}\right)\right) h \cdot f=-2 \mathrm{i} \bar{k} \bar{k}, & \left(D_{x}^{2}+\mathrm{i}\left(D_{t}-D_{y}\right)\right) \bar{h} \cdot f=-2 i g k, \\
D_{x} D_{t} f \cdot f=-2(g \bar{g}+h \bar{h}), \quad D_{x} k \cdot f=-\bar{g} \bar{h}, \quad D_{x} \bar{k} \cdot f=g h .
\end{array}
$$


Consider solutions satisfying the following condition:

$$
\bar{g} \mathcal{G}=(g \mathcal{G})^{*}, \quad \bar{h} \mathcal{G}=(h \mathcal{G})^{*}, \quad \bar{k} \mathcal{G}=-(k \mathcal{G})^{*}, \quad f \mathcal{G}: \text { real }
$$

where $\mathcal{G}$ is a gauge factor. Then, for $F=f \mathcal{G}, G=g \mathcal{G}, H=h \mathcal{G}$ and $K=k \mathcal{G}$, we will obtain the bilinear equations of the 2c-2d-LSRI system (4). Thus, the 2c-2d-LSRI system has a three-component Wronskian solution.

To satisfy the condition (5), we consider the following constrained case: $N=M+$ $L, \psi_{i}=0$ for $2 M+1 \leq i \leq 2 M+2 L, \chi_{i}=0$ for $1 \leq i \leq 2 M$ and

$$
\begin{aligned}
& \varphi_{i}=e^{\xi_{i}}, \quad \varphi_{M+i}=e^{-\xi_{i}^{*}}, \quad \xi_{i}=p_{i} x_{1}+p_{i}^{2} x_{2}, \\
& \psi_{i}=a_{i} e^{\eta_{i}}, \quad \psi_{M+i}=a_{M+i} e^{-\eta_{i}^{*}}, \quad \eta_{i}=q_{i} y_{1}+\eta_{i 0},
\end{aligned}
$$

for $i=1,2, \ldots, M$, and

$$
\begin{aligned}
& \varphi_{2 M+i}=e^{\theta_{i}}, \quad \varphi_{2 M+L+i}=e^{-\theta_{i}^{*}}, \quad \theta_{i}=s_{i} x_{1}+s_{i}^{2} x_{2}, \\
& \chi_{2 M+i}=b_{i} e^{\zeta_{i}}, \quad \chi_{2 M+L+i}=b_{L+i} e^{-\zeta_{i}^{*}}, \quad \zeta_{i}=r_{i} z_{1}+\zeta_{i 0},
\end{aligned}
$$

for $i=1,2, \ldots, L$, where $p_{i}, s_{i}, q_{i}$ and $r_{i}$ are wave numbers and $\eta_{i 0}$ and $\zeta_{i 0}$ are phase constants. The parameters $a_{i}$ and $b_{i}$ must be determined from the condition of complex conjugacy. By using the standard technique [6], $a_{i}$ and $b_{i}$ are determined as

$$
\begin{aligned}
& a_{i}=\prod_{\substack{k=1 \\
k \neq i}}^{M} \frac{p_{k}-p_{i}}{q_{k}-q_{i}} \prod_{k=1}^{M} \frac{p_{k}^{*}+p_{i}}{q_{k}^{*}+q_{i}}, \quad a_{M+i}=\prod_{k=1}^{L}\left(s_{k}+p_{i}^{*}\right)\left(s_{k}^{*}-p_{i}^{*}\right), \quad 1 \leq i \leq M, \\
& b_{i}=\prod_{\substack{k=1 \\
k \neq i}}^{L} \frac{s_{k}-s_{i}}{r_{k}-r_{i}} \prod_{k=1}^{L} \frac{s_{k}^{*}+s_{i}}{r_{k}^{*}+r_{i}}, \quad b_{L+i}=\prod_{k=1}^{M}\left(p_{k}+s_{i}^{*}\right)\left(p_{k}^{*}-s_{i}^{*}\right), \quad 1 \leq i \leq L,
\end{aligned}
$$

and condition (5) is satisfied for the gauge factor,

$$
\begin{aligned}
\mathcal{G} & =\prod_{1 \leq i<j \leq M}\left(p_{j}^{*}-p_{i}^{*}\right)\left(q_{i}-q_{j}\right) \prod_{1 \leq i<j \leq L}\left(s_{j}^{*}-s_{i}^{*}\right)\left(r_{i}-r_{j}\right) \prod_{i=1}^{M} \prod_{j=1}^{L}\left(p_{i}-s_{j}\right) \\
& \times e^{\sum_{i=1}^{M}\left(\xi_{i}^{*}-\eta_{i}\right)+\sum_{j=1}^{L}\left(\theta_{j}^{*}-\zeta_{j}\right)} .
\end{aligned}
$$

This solution represents the $(M+L)$-soliton, i.e., $M$ solitons propagate on the first component of short wave $S^{(1)}$ whose complex wave numbers are given by $p_{i}$ and $q_{i}$ and complex phase constants are $\eta_{i 0}$ and $L$ solitons propagate on the second one $S^{(2)}$ whose complex wave numbers and phase constants are $s_{i}, r_{i}$ and $\zeta_{i 0}$.

For instance by taking $M=L=1,(1+1)$-soliton solution is given as

$$
\begin{aligned}
F= & f \mathcal{G}=c\left(\frac{p+p^{*}}{q+q^{*}} \frac{s+s^{*}}{r+r^{*}} \frac{1}{\left|p+s^{*}\right|^{2}}-\frac{s+s^{*}}{r+r^{*}} e^{\xi+\xi^{*}-\eta-\eta^{*}}-\frac{p+p^{*}}{q+q^{*}} e^{\theta+\theta^{*}-\zeta-\zeta^{*}}\right. \\
& \left.+|p-s|^{2} e^{\xi+\xi^{*}-\eta-\eta^{*}+\theta+\theta^{*}-\zeta-\zeta^{*}}\right), \\
G= & g \mathcal{G}=c\left(p+p^{*}\right) e^{\xi-\eta}\left(\frac{s+s^{*}}{r+r^{*}} \frac{1}{p^{*}+s}-(p-s) e^{\theta+\theta^{*}-\zeta-\zeta^{*}}\right),
\end{aligned}
$$



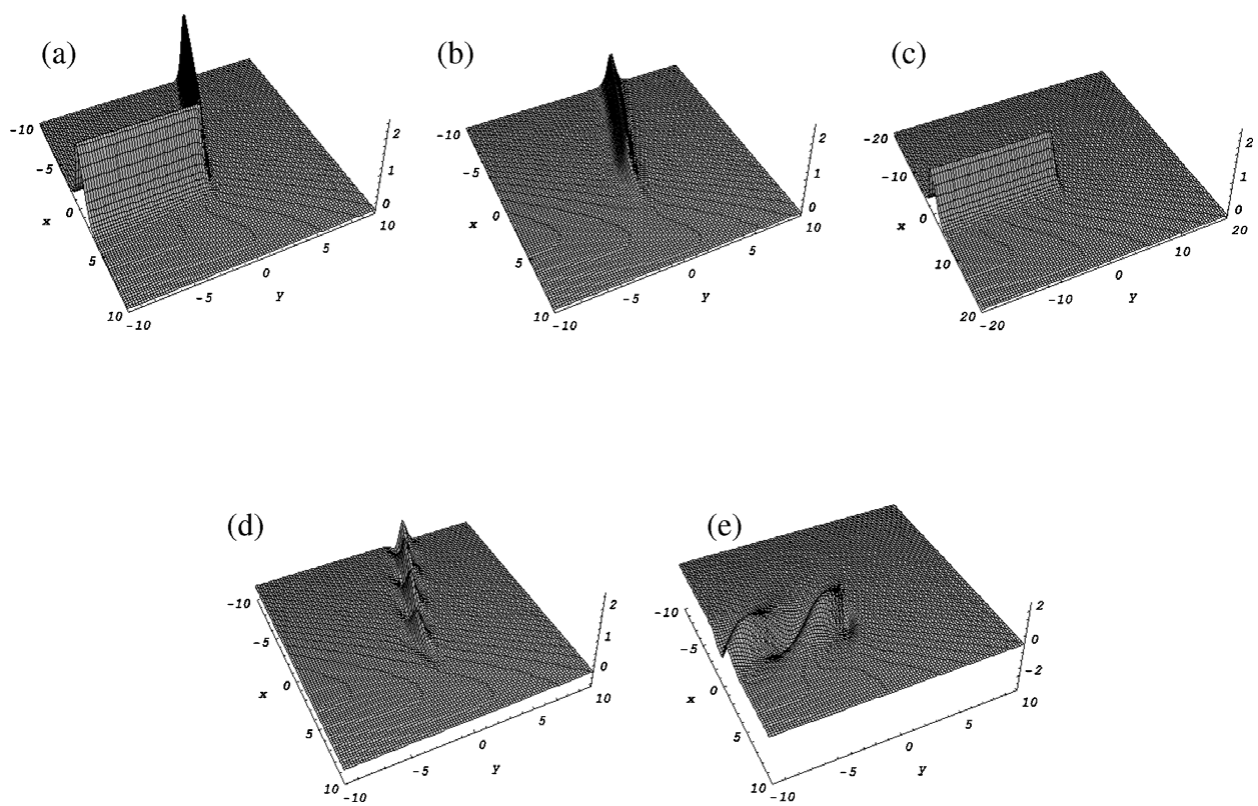

Figure 1. Single-line soliton of equations (2), which is obtained by tau-functions of (6). (a) $-L$, (b) $\left|S^{(1)}\right|$, (c) $\left|S^{(2)}\right|$, (d) $\operatorname{Re}\left[S^{(1)}\right]$, (e) $\operatorname{Re}\left[S^{(2)}\right]$. The parameters are $p=1+\mathrm{i}, q=-1+2 \mathrm{i}, r=-2+\mathrm{i}$.

$$
\begin{aligned}
& H=h \mathcal{G}=-c\left(s+s^{*}\right) e^{\theta^{*}-\zeta^{*}}\left(\frac{p+p^{*}}{q+q^{*}} \frac{1}{p^{*}+s}+\left(p^{*}-s^{*}\right) e^{\xi+\xi^{*}-\eta-\eta^{*}}\right), \\
& K=k \mathcal{G}=c \frac{\left(p+p^{*}\right)\left(s+s^{*}\right)}{p+s^{*}} e^{\xi^{*}-\eta^{*}+\theta-\zeta},
\end{aligned}
$$

where $c=-\left|(p-s)\left(p+s^{*}\right)\right|^{2}$ and we dropped the index 1 for simplicity. In order to satisfy the regularity condition $F \neq 0$, we can take $\operatorname{Re} p>0, \operatorname{Re} s>0, \operatorname{Re} q<0$ and $\operatorname{Re} r<0$. After removing the gauge and constant factors, by choosing the same wave number in $x$-direction for the above two solitons, i.e. $s=p$, we obtain the single soliton solution,

$$
\begin{aligned}
& F=\frac{1}{p+p^{*}}-e^{\xi+\xi^{*}}\left(\left(q+q^{*}\right) e^{-\eta-\eta^{*}}+\left(r+r^{*}\right) e^{-\zeta-\zeta^{*}}\right), \\
& G=\left(q+q^{*}\right) e^{\xi-\eta}, \quad H=-\left(r+r^{*}\right) e^{\xi^{*}-\zeta^{*}}, \quad K=\left(q+q^{*}\right)\left(r+r^{*}\right) e^{\xi+\xi^{*}-\eta^{*}-\zeta},
\end{aligned}
$$

where $\xi=p x-i p^{2} y, \eta=q(y-t)+\eta_{0}$ and $\zeta=-r(y+t)+\zeta_{0}$. Figure 1 . shows the plots of this single soliton solution. $L$ shows V-shape soliton, $\left|S^{(1)}\right|$ and $\left|S^{(2)}\right|$ show solitoff behaviour [5].

3. Solutions in the case without $Q$. We consider the 2c-2d-LSRI system (1) without the fourth field $Q$ in (2). This system (1) describes waves in the two-layer fluid. Setting $L=-(2 \log F)_{x x}, S^{(1)}=G / F, S^{(2)}=H / F$, we have

$$
\begin{aligned}
& {\left[\mathrm{i}\left(D_{t}+D_{y}\right)-D_{x}^{2}\right] G \cdot F=0, \quad\left[\mathrm{i}\left(D_{t}-D_{y}\right)-D_{x}^{2}\right] H \cdot F=0,} \\
& -\left(D_{t} D_{x}-2 c\right) F \cdot F=2 G G^{*}+2 H H^{*} .
\end{aligned}
$$


Here we consider the case of $c=0$.

Using the procedure of the Hirota bilinear method, we obtain the single soliton solution

$$
\begin{gathered}
F=1+A_{11} \exp \left(\eta_{1}+\eta_{1}^{*}\right), \quad G=a_{1} \exp \left(\eta_{1}\right), \quad H=b_{1} \exp \left(\xi_{1}\right), \\
\eta_{1}=p_{1} x+\mathrm{i} q_{1} y+\lambda_{1} t+\eta_{1}^{(0)}, \quad \xi_{1}=p_{1} x-\mathrm{i} q_{1} y+\lambda_{1} t+\eta_{1}^{(0)}, \\
A_{11}=-\frac{a_{1} a_{1}^{*}+b_{1} b_{1}^{*}}{\left(p_{1}+p_{1}^{*}\right)\left(\lambda_{1}+\lambda_{1}^{*}\right)}, \quad \lambda_{1}=-\mathrm{i} p_{1}^{2}-\mathrm{i} q_{1} .
\end{gathered}
$$

Here, $q_{1}$ is a real number. We can rewrite $A_{11}$ as

$$
A_{11}=-\frac{a_{1} a_{1}^{*}+b_{1} b_{1}^{*}}{\left(p_{1}+p_{1}^{*}\right)^{2}\left(\mathrm{i} p_{1}^{*}-\mathrm{i} p_{1}\right)} .
$$

Thus, we have

$$
\begin{aligned}
S^{(1)} & =\frac{a_{1} \exp \left(\eta_{1}\right)}{1+A_{11} \exp \left(\eta_{1}+\eta_{1}^{*}\right)}, \quad S^{(2)}=\frac{b_{1} \exp \left(\xi_{1}\right)}{1+A_{11} \exp \left(\eta_{1}+\eta_{1}^{*}\right)}, \\
L & =-2 \frac{\partial^{2}}{\partial x^{2}} \log \left(1+A_{11} \exp \left(\eta_{1}+\eta_{1}^{*}\right)\right) .
\end{aligned}
$$

Since $\left|S^{(1)}\right|^{2}=G G^{*} / F^{2},\left|S^{(2)}\right|^{2}=H H^{*} / F^{2}, L=-(2 \log F)_{x x}$ do not include $y$, all solitons propagate in the $x$-direction.

There is an exact solution depending on $y$-variable,

$$
\begin{aligned}
& S^{(1)}=\frac{A_{1} \exp (p x+q y+r t)}{1+\exp (2(p x+q y+r t))} \exp \left(\mathrm{i}\left(k_{1} x+l_{1} y+m_{1} t\right)\right), \\
& S^{(2)}=\frac{A_{2} \exp (p x+q y+r t)}{1+\exp (2(p x+q y+r t))} \exp \left(\mathrm{i}\left(k_{2} x+l_{2} y+m_{2} t\right)\right), \\
& L=\frac{A \exp (2(p x+q y+r t))}{(1+\exp (2(p x+q y+r t)))^{2}},
\end{aligned}
$$

where $p, q, r, k_{1}, l_{1}, m_{1}, k_{2}, l_{2}, m_{2}, A_{1}, A_{2}$ and $A$ satisfy the relations $r=\left(k_{1}+k_{2}\right) p, q=\left(k_{1}-k_{2}\right) p, m_{1}=k_{1}^{2}-l_{1}-p^{2}, m_{2}=k_{2}^{2}+l_{2}-p^{2}, A=-8 p^{2}, A_{1}^{2}+$ $A_{2}^{2}=-4\left(k_{1}+k_{2}\right) p^{2}$, and $p, q, k_{1}, l_{1}, l_{2}$ are arbitrary parameters. In Figure 2., we see that waves in $S^{(1)}$ and $S^{(2)}$ have different modulation property, i.e. carrier waves in $S^{(1)}$ and $S^{(2)}$ has different directions of propagation. Note that the solutions of equations (2) also have this property.

It seems that equations (1) are non-integrable and do not admit general $N$-soliton solution. Similar system (2) has an $N$-soliton solution, but its physical derivation has not been done yet.

4. Concluding remarks. We have studied solutions of a new integrable $2 \mathrm{c}-2 \mathrm{~d}$ LSRI system (2). We presented a Wronskian formula for 2c-2d LSRI system (2) with complex conjugacy condition. We have also presented solutions of the system (1) in the case of two-layer fluid, i.e. the $2 \mathrm{c}-2 \mathrm{~d}$ LSRI system without $Q$. In this case, the system 

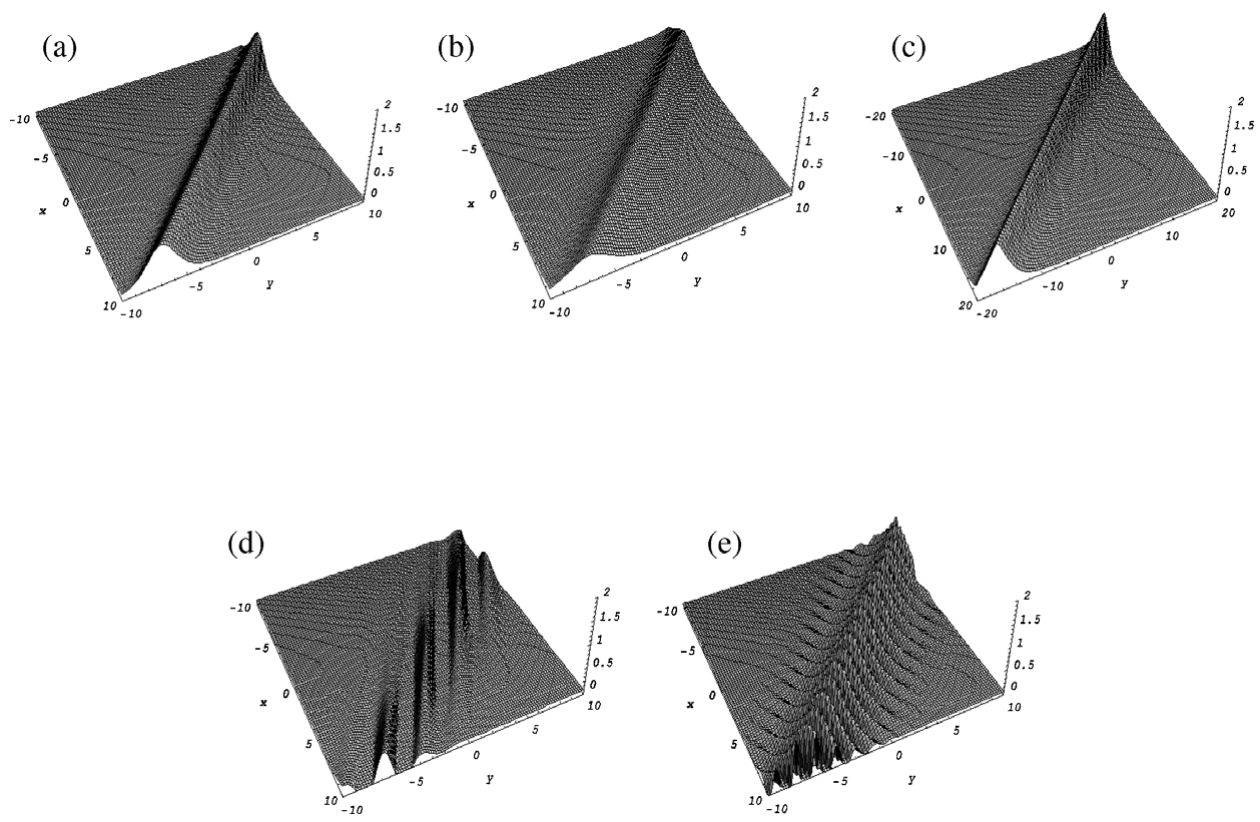

Figure 2. Line soliton of equations (1). (a) $-L$, (b) $\left|S^{(1)}\right|$, (c) $\left|S^{(2)}\right|$, (d) $\operatorname{Re}\left[S^{(1)}\right]$, (e) $\operatorname{Re}\left[S^{(2)}\right]$. The parameters are $k_{1}=-1, k_{2}=-2, A_{1}=1, A_{2}=2, l_{1}=3, l_{2}=4$.

(1) seems to be non-integrable, i.e. the system (1) does not have multi-soliton solutions. We have found that waves in $S^{(1)}$ and $S^{(2)}$ in both systems have different modulation property, i.e. carrier waves in $S^{(1)}$ and $S^{(2)}$ have different directions of propagation. But system (2) has much more interesting solutions such as the V-shape soliton and solitoff because of integrability.

ACKNOWLEDGEMENTS. One of the authors (Ken-ichi Maruno) wishes to acknowledge organizers for providing the financial support for the ISLAND 3 (Integrable Systems: Linear And Nonlinear Dynamics 3) conference.

\section{REFERENCES}

1. M. J. Ablowitz, B. Prinari and A. D. Trubatch, Discrete and continuous nonlinear Schrödinger systems (Cambridge University Press, Cambridge, UK, 2004).

2. M. J. Ablowitz, B. Prinari and A. D. Trubatch, Soliton interactions in the vector NLS equation, Inv. Probl. 20 (2004), 1217-1237.

3. E. Date, M. Jimbo, M. Kashiwara and T. Miwa, Transformation groups for soliton equations. III. Operator approach to the Kadomtsev-Petviashvili equation, J. Phys. Soc. Jpn. 50 (1981), 3806-3812.

4. E. Date, M. Jimbo, M. Kashiwara and T. Miwa, Transformation group for soliton equations: Euclidean Lie algebras and reduction of the KP hierarchy, Publ. Res. Inst. Math. Sci. 18 (1982), 1077-1111.

5. C. R. Gilson, Resonant behaviour in the Davey-Stewartson equation, Phys. Lett. A 161 (1992), 423-428.

6. J. Hietarinta and R. Hirota, Multidromion solutions to the Davey-Stewartson equation, Phys. Lett. A 145 (1990), 237-244.

7. R. Hirota, The direct method in soliton theory (Cambridge University Press, Cambridge, UK, 2004). 
8. S. V. Manakov, On the theory of two-dimensional stationary self-focusing of electromagnetic waves, Sov. Phys. JETP 38 (1974), 248-253.

9. M. Oikawa, Y. Ohta and K. Maruno, Long wave-short wave resonance interaction system, Reports of RIAM Symposium No. 18ME-S5, 2007.

10. M. Oikawa, M. Okamura and M. Funakoshi, Two-dimensional resonant interaction between long and short waves, J. Phys. Soc. Jpn. 58 (1989), 4416-4430.

11. Y. Ohta, K. Maruno and M. Oikawa, Two-component analogue of two-dimensional long wave-short wave resonance interaction equations: A derivation and solutions, J. Phys. A: Math. Theor. 40 (2007), 7659-7672.

12. M. Onorato, A. R. Osborne and M. Serio, Modulational instability in crossing sea states: A possible mechanism for the formation of freak waves, Phys. Rev. Lett. 96 (2006), 014503-1-014503-4.

13. R. Radhakrishnan, M. Lakshmanan and J. Hietarinta, Inelastic collision and switching of coupled bright solitons in optical fibers, Phys. Rev. E 56 (1997), 2213-2216.

14. N. Yajima and M. Oikawa, Formation and interaction of sonic-Langmuir solitons inverse scattering method, Prog. Theor. Phys. 56 (1976), 1719-1739. 